\title{
Plurigenera of general type surfaces in mixed characteristic
}

\author{
Junecue Suh
}

\begin{abstract}
We construct general type surfaces in mixed characteristic whose geometric genera can be made to jump by an arbitrarily prescribed positive amount under specialization. We then show that this phenomenon of jumping geometric genus presents itself in some compact Shimura surfaces. Finally, we find a set of conditions, met by the latter Shimura surfaces, that forces the higher plurigenera to remain constant in reduction modulo $p$.
\end{abstract}

\section{Introduction}

In equal characteristic zero, it has been proven by Iitaka (for surfaces), by Kollár-Mori (for threefolds) and by Siu (in arbitrary dimension) that the plurigenus

$$
P_{m}(X / K)=\operatorname{dim}_{K} H^{0}\left(X, \omega_{X / K}^{\otimes m}\right), \quad \text { for } m \geqslant 1
$$

of a projective smooth variety $X / K$ (where $\omega_{X / K}$ stands for the canonical bundle) is invariant under deformation.

On the other hand, in mixed characteristic $(0, p)$ or in equal characteristic $p$, where $p$ is a prime number, $P_{m}$ is no longer invariant under deformation; in [Lan83, Theorem 1.4], Lang shows that a $\mu_{2}$ Enriques surface in characteristic two, whose geometric genus is one, lifts algebraically to the Witt vectors, and that the generic fibre of the lifting (which can also be shown to be an Enriques surface) has geometric genus zero; and in [KU85, Examples 8.7 and 8.8], Katsura and Ueno construct (a) a deformation of an elliptic surface in equal characteristic $p$ and (b) a lifting of an elliptic surface in characteristic $p>0$ to characteristic zero (where one allows tame ramification above $p$ ), in which fibres have different plurigenera.

In this article, we first construct surfaces of general type over any discrete valuation ring of mixed characteristic $(0, p)$ whose fibres have any possible prescribed gap in geometric genera. More precisely, we have the following.

Theorem 0.1. Let $R$ be a discrete valuation ring with residue class field $k$ of characteristic $p>0$ and with fraction field $K$ of characteristic zero. Given any integer $e \geqslant 1$, there exists a projective smooth scheme $Y / R$ of relative dimension $d=2$ (respectively $d \geqslant 2$ ) with geometrically connected fibres such that

$$
\begin{aligned}
p_{g}\left(Y_{k} / k\right) & =p_{g}\left(Y_{K} / K\right)+e \\
\left(\text { respectively } p_{g}\left(Y_{k} / k\right)\right. & \left.\geqslant p_{g}\left(Y_{K} / K\right)+e\right),
\end{aligned}
$$

where $p_{g}=P_{1}$ stands for the geometric genus. Moreover, the fibres may be assumed to have ample canonical bundles.

Received 8 August 2007, accepted in final form 9 January 2008, published online 22 July 2008.

2000 Mathematics Subject Classification 14L30, 14J29, 14 G35.

Keywords: algebraic surface, plurigenus, mixed characteristic.

This journal is (C) Foundation Compositio Mathematica 2008. 


\section{PluRigenera of General tyPe SURFACES IN MiXed CHARACTERISTIC}

Next we show that some compact Shimura varieties exhibit this phenomenon.

TheOREM. Let $X$ be a connected component of a quaternionic or unitary Shimura surface of the type considered in $\S 3.1$. Then for any finite set of rational prime numbers $p_{1}, \ldots, p_{n}$ satisfying the conditions in $\S 3.2$ and for any integer $e \geqslant 0$, there exists a finite étale cover $Y$ (which is a Shimura variety of the same type as $X$ ) of $X$, defined over a number field $E_{Y}$, such that for any prime $\wp$ of $E_{Y}$ dividing $p_{i}(i=1, \ldots, n), Y$ has good reduction $Y_{\wp}$ at $\wp$ and one has

$$
p_{g}\left(Y_{\wp}\right) \geqslant p_{g}(Y)+e .
$$

Finally, we find some conditions under which the plurigenera remain the same in reduction modulo $p$; see Theorem 1.2.1 and Corollary 1.2.4.

\section{Notation}

The following notation is used in the first two sections. We denote by $p$ a prime number and by $R$ a discrete valuation ring of mixed characteristic $(0, p)$, with $k$ (respectively $K$ ) its residue class field (respectively its fraction field). For any scheme $Z$ over $R$, we denote by $Z_{k}$ (respectively $Z_{K}$ ) its special fibre $Z \otimes_{R} k$ (respectively its generic fibre $Z \otimes_{R} K$ ). Similarly, for any $R$-morphism $f: Z^{\prime} \longrightarrow Z$, we denote by $f_{k}: Z_{k}^{\prime} \longrightarrow Z_{k}$ (respectively $f_{K}: Z_{K}^{\prime} \longrightarrow Z_{K}$ ) its base change by $R \longrightarrow k$ (respectively $R \longrightarrow K$ ).

For any field $F$ and a proper smooth variety $X / F$, we denote by

$$
P_{m}(X / F)=\operatorname{dim}_{F} H^{0}\left(X, \omega_{X / F}^{\otimes m}\right)
$$

the $m$ th plurigenus of $X / F$, where $\omega_{X / F}$ denotes the canonical line bundle (the highest exterior power of the sheaf of Kähler differentials). Finally, put

$$
p_{g}(X / F)=P_{1}(X / F) .
$$

\section{Group cohomology and plurigenera of general type surfaces}

\subsection{Group cohomology and geometric genus}

Let us fix an integer $e \geqslant 1$ and put $G:=(\mathbb{Z} / p \mathbb{Z})^{\oplus e}$, the $e$-fold direct product of the finite group $\mathbb{Z} / p \mathbb{Z}$. Recall that if $F$ is a field of characteristic different from $p$, then the group cohomology ring $H^{*}(G, F)$, where $F$ is equipped with the trivial action, is concentrated in degree 0 , where it is $F$. In contrast, if char $F=p$, then $H^{*}(G, F)$, with trivial $G$-action on $F$, is isomorphic either to the polynomial ring $F\left[x_{1}, \ldots, x_{n}\right]$ with $\operatorname{deg} x_{i}=1$ in case $p=2$, or to the tensor product of the polynomial ring $F\left[y_{1}, \ldots, y_{n}\right]$ and the exterior algebra $\bigwedge^{*}\left(z_{1}, \ldots, z_{n}\right)$, with $\operatorname{deg} y_{i}=2$ and $\operatorname{deg} z_{i}=1$, in case $p \geqslant 3$ (see, for example, [AM94, Corollary II.4.3 and Theorem II.4.4]).

LEMma 1.1.1. Let $X / R$ be a projective smooth scheme of relative dimension $d$ with geometrically connected and nonempty fibres, equipped with a free $R$-morphic action of $G$. Denote by $Y / R$ the quotient of $X / R$ by $G$.

(i) Suppose $d=2$ and $H^{1}\left(X_{K}, \mathcal{O}_{X_{K}}\right)=0$. Then

$$
p_{g}\left(Y_{k} / k\right) \geqslant p_{g}\left(Y_{K} / K\right)+e .
$$

If, in addition, we assume $H^{1}\left(X_{k}, \mathcal{O}_{X_{k}}\right)=0$, then we actually have equality.

(ii) Suppose $d \geqslant 2$ and

$$
H^{i}\left(X_{K}, \mathcal{O}_{X_{K}}\right)=0=H^{i}\left(X_{k}, \mathcal{O}_{X_{k}}\right) \quad \text { for every } 0<i<d .
$$




\section{J. SuH}

Then $p_{g}\left(Y_{k} / k\right)-p_{g}\left(Y_{K} / K\right)$ is equal to

$$
G_{d}(e)=(-1)^{d-1} \sum_{i=1}^{d-1}(-1)^{i} \operatorname{dim} H^{i}(G, k),
$$

which, for a fixed $d \geqslant 2$, is a polynomial in $e$ of degree $d-1$ with positive leading coefficient. In particular, $G_{d}(e) \rightarrow \infty$ as $e \rightarrow \infty$.

Proof. (i) By the assumptions on $X / R$, we have

$$
H^{0}\left(X_{K}, \mathcal{O}_{X_{K}}\right)=K \quad \text { and } \quad H^{0}\left(X_{k}, \mathcal{O}_{X_{k}}\right)=k
$$

Therefore, the Serre-Hochschild spectral sequence gives

$$
H^{0}\left(Y_{K}, \mathcal{O}_{Y_{K}}\right)=K, \quad H^{0}\left(Y_{k}, \mathcal{O}_{Y_{k}}\right)=k \quad \text { and } \quad H^{1}\left(Y_{K}, \mathcal{O}_{Y_{K}}\right)=0,
$$

together with an exact sequence

$$
0 \longrightarrow H^{1}\left(G, H^{0}\left(X_{k}, \mathcal{O}_{X_{k}}\right)\right) \longrightarrow H^{1}\left(Y_{k}, \mathcal{O}_{Y_{k}}\right) \longrightarrow H^{1}\left(X_{k}, \mathcal{O}_{X_{k}}\right)^{G}
$$

Thus, we obtain $\operatorname{dim}_{k} H^{1}\left(Y_{k}, \mathcal{O}_{Y_{k}}\right) \geqslant \operatorname{dim} H^{1}(G, k)=e$; when $H^{1}\left(X_{k}, \mathcal{O}_{X_{k}}\right)$ vanishes, one even obtains an equality.

As $Y / R$ is smooth, hence flat, we have [EGAIII, Théorème 7.9.4]

$$
\chi\left(Y_{K}, \mathcal{O}_{Y_{K}}\right)=\chi\left(Y_{k}, \mathcal{O}_{Y_{k}}\right),
$$

which implies

$$
\operatorname{dim}_{k} H^{2}\left(Y_{k}, \mathcal{O}_{Y_{k}}\right)=\operatorname{dim}_{K} H^{2}\left(Y_{K}, \mathcal{O}_{Y_{K}}\right)+\operatorname{dim}_{k} H^{1}\left(Y_{k}, \mathcal{O}_{Y_{k}}\right) .
$$

We conclude by Serre duality.

(ii) The Serre-Hochschild spectral sequence gives, for each $0<i<d$,

$$
H^{i}\left(Y_{K}, \mathcal{O}_{Y_{K}}\right)=0 \quad \text { while } H^{i}\left(Y_{k}, \mathcal{O}_{Y_{k}}\right) \cong H^{i}(G, k) .
$$

The invariance of Euler characteristic and Serre duality imply the equality above. It follows from the explicit description of $H^{*}(G, k)$ recalled above that $G_{d}(e)$ has the asserted property.

This lemma will be applied in the following sections in order to construct quotients where the geometric genus jumps. Before that, we turn to higher $(m \geqslant 2)$ plurigenera $P_{m}$ of general type surfaces.

\subsection{Plurigenera of general type surfaces in mixed characteristic}

TheOREM 1.2.1. Let $R$ be a discrete valuation ring whose fraction field $K$ (respectively residue field $k$ ) has characteristic zero (respectively is perfect of characteristic $p>0$ ) and let $X / R$ be a proper smooth algebraic space of relative dimension two.

(i) One has

$$
p_{g}\left(X_{k}\right)-p_{g}\left(X_{K}\right)=\operatorname{dim} \operatorname{Tgt}_{0}\left(\operatorname{Pic}_{X_{k} / k}\right)-\operatorname{dim} \operatorname{Pic}_{X_{k} / k} .
$$

In particular, $p_{g}\left(X_{k}\right)=p_{g}\left(X_{K}\right)$ if and only if $\mathrm{Pic}_{X_{k} / k}$ is reduced.

(ii) If $X_{k}$ lifts to $W_{2}(k)$ and is of general type, then one has

$$
P_{m}\left(X_{K}\right)=P_{m}\left(X_{k}\right) \quad \text { for every integer } m \geqslant 2 \text {. }
$$

Proof. By extending $R$ if necessary, we may and do assume that $R$ is complete and that $k$ is algebraically closed. 


\section{PluRigenera of General TYPE SURFACES IN MIXED CHARACTERISTIC}

(i) By Serre duality and invariance of Euler characteristic (cf. Lemma 1.1.1), we have

$$
\begin{aligned}
p_{g}\left(X_{k}\right)-p_{g}\left(X_{K}\right) & =\operatorname{dim}_{k} H^{1}\left(X_{k}, \mathcal{O}_{X_{k}}\right)-\operatorname{dim}_{k} H^{1}\left(X_{K}, \mathcal{O}_{X_{K}}\right) \\
& =\operatorname{dim}_{0} \operatorname{Tgt}_{0}\left(\operatorname{Pic}_{X_{k} / k}\right)-\operatorname{dim}_{0} \operatorname{Tgt}_{0}\left(\operatorname{Pic}_{X_{K} / K}\right) .
\end{aligned}
$$

As $K$ has characteristic zero and as $X / R$ has geometrically normal fibres, we conclude by (the translation in terms of algebraic spaces of) [Gro62, Théorème 2.1(ii) and Corollaire 2.7].

(ii) By [KU85, Lemmas 9.4 and 9.6], after passing to an extension of $R$, we get a proper smooth algebraic space $X^{\text {min }} / R$ with an $R$-morphism $X \longrightarrow X^{\text {min }}$ such that (i) both $X_{k} \longrightarrow X_{k}^{\min }$ and $X_{K} \longrightarrow X_{K}^{\min }$ are obtained by successive blow-downs of $(-1)$-curves and (ii) $X_{k}^{\min }$ and $X_{K}^{\min }$ are minimal (thus, their canonical bundles are big and numerically effective). The next proposition shows that $X_{k}^{\min } / k$ lifts to $W_{2}(k)$.

Proposition 1.2.2. Let $Y$ be a proper smooth surface over a field $k$ and let $A$ be an Artin local ring with residue field $k$ and with square-zero maximal ideal $\underline{\mathrm{m}}$. Consider a $k$-rational point $p \in Y(k)$ and the blow-up $Y^{\prime}:=B l_{p}(Y)$. Then the obstruction class

$$
o(Y / k / A) \in H^{2}\left(Y, \underline{\mathrm{m}} \otimes_{k} T_{Y / k}\right)
$$

to the existence of a flat lifting $\tilde{Y} / A$ of $Y / k$ vanishes if and only if the analogous class $o\left(Y^{\prime} / k / A\right) \in$ $H^{2}\left(Y^{\prime}, \underline{\mathrm{m}} \otimes_{k} T_{Y^{\prime} / k}\right)$ does.

Proof. Let $\pi: Y^{\prime} \longrightarrow Y$ denote the blow-up and $i: E \hookrightarrow Y^{\prime}$ the inclusion of the exceptional divisor. Then we have an exact sequence

$$
0 \longrightarrow \pi^{*} \Omega_{Y / k}^{1} \longrightarrow \Omega_{Y^{\prime} / k}^{1} \longrightarrow i_{*} \Omega_{E / k}^{1} \longrightarrow 0 .
$$

Taking $R \underline{\operatorname{Hom}}\left(-, \mathcal{O}_{Y^{\prime}}\right)$, we get an exact triangle

$$
R \underline{\operatorname{Hom}}_{\mathcal{O}_{Y^{\prime}}}\left(i_{*} \Omega_{E / k}^{1}, \mathcal{O}_{Y^{\prime}}\right) \longrightarrow T_{Y^{\prime} / k} \longrightarrow \pi^{*} T_{Y / k} \stackrel{+1}{\longrightarrow} .
$$

By Grothendieck duality, the first term is isomorphic to

$$
\begin{aligned}
i_{*} R \underline{\operatorname{Hom}}_{\mathcal{O}_{E}}\left(\Omega_{E / k}^{1}, R i ! \mathcal{O}_{Y^{\prime}}\right) & \cong i_{*}\left(T_{E / k} \otimes R i i^{\prime} \mathcal{O}_{Y^{\prime}}\right) \\
& \cong i_{*}\left(T_{E / k} \otimes \mathcal{N}_{E / Y^{\prime}}\right)[-1],
\end{aligned}
$$

where $\mathcal{N}_{E / Y^{\prime}}$ denotes the normal bundle of $E$ in $Y^{\prime}$, whose degree is equal to

$$
\operatorname{deg}_{E} \mathcal{N}_{E / Y^{\prime}}=E^{2}=-1 .
$$

As $\operatorname{deg}_{E} T_{E / k}=2$, the first term of (1) is isomorphic to $i_{*} \mathcal{O}_{E}(1)[-1]$. The long exact cohomology sequence now gives an exact sequence

$$
H^{1}\left(E, \mathcal{O}_{E}(1)\right) \longrightarrow H^{2}\left(Y^{\prime}, T_{Y^{\prime} / k}\right) \longrightarrow H^{2}\left(Y^{\prime}, \pi^{*} T_{Y / k}\right) \longrightarrow 0,
$$

hence, the natural morphism

$$
H^{2}\left(Y^{\prime}, \underline{\mathrm{m}} \otimes_{k} T_{Y^{\prime} / k}\right) \longrightarrow H^{2}\left(Y^{\prime}, \underline{\mathrm{m}} \otimes_{k} \pi^{*} T_{Y / k}\right)
$$

is an isomorphism. On the other hand, by the projection formula, we have

$$
R \pi_{*} \pi^{*} T_{Y / k} \cong T_{Y / k} \otimes_{\mathcal{O}_{Y}} R \pi_{*} \mathcal{O}_{Y^{\prime}} \cong T_{Y / k},
$$

hence, an identification of three cohomology groups

$$
H^{2}\left(Y^{\prime}, \underline{\mathrm{m}} \otimes T_{Y^{\prime} / k}\right) \stackrel{\sim}{\longrightarrow} H^{2}\left(Y^{\prime}, \underline{\mathrm{m}} \otimes \pi^{*} T_{Y / k}\right) \stackrel{\sim}{\sim} H^{2}\left(Y, \underline{\mathrm{m}} \otimes T_{Y / k}\right),
$$

via natural morphisms. We conclude with [Ill05, Remark 8.5.10(a)]. 


\section{J. SuH}

Coming back to the proof of the theorem, we are reduced to the case where both $X_{k}$ and $X_{K}$ are minimal (note that $P_{m}$ is a birational invariant). Then by Kodaira vanishing [DI87, Corollaire 2.8],

$$
P_{m}\left(X_{k}\right)=\chi\left(X_{k}, \omega_{X_{k}}^{\otimes m}\right)=\chi\left(X_{K}, \omega_{X_{K}}^{\otimes m}\right)=P_{m}\left(X_{K}\right)
$$

for every integer $m \geqslant 2$.

Remark 1.2.3. When $R$ has equal characteristic $p>0$, a similar argument proves the invariance of higher plurigenera of general type surfaces, assuming that both geometric fibres lift to $W_{2}$.

Corollary 1.2.4. Let $X$ and $R$ be as in Theorem 1.2.1. If the special fibre $X_{k}$ :

(i) lifts to $W_{2}(k)$ (automatic if $R$ is unramified);

(ii) has reduced Picard scheme; and

(iii) is of general type;

then we have

$$
P_{m}\left(X_{K}\right)=P_{m}\left(X_{k}\right), \quad \text { for every } m \geqslant 1 \text {. }
$$

\section{Finite quotients of complete intersections}

In this section, we construct complete intersections with free $(\mathbb{Z} / p)^{\oplus e}$ actions, so that Lemma 1.1.1 can be applied to yield Theorem 0.1 .

The idea of using complete intersections with finite group actions to construct pathological examples goes back to Godeaux and Serre [Ser58]. A more general situation, where the base can be a noetherian complete local ring and the group (scheme) is allowed to have infinitesimal part, was considered by Raynaud in [Ray79, Proposition 4.2.3] which can then be used to construct, among other things, surfaces with non-reduced Picard schemes; in view of Theorem 1.2.1, these surfaces have jumping geometric genus.

We recall the construction, with more focus on the explicit number of variables and degrees involved. This is used when we analyze the Kodaira dimension in $\S 2.4$. The analysis shows, in essence, that the examples in Theorem 0.1, with the exception of $p=2$ and $e^{\prime}=1$ (considered in $\S 2.5)$, necessarily have ample canonical bundles.

\subsection{Complete intersection with free $G$-action}

Lemma 2.1.1. Let $\rho: G \longrightarrow \operatorname{PGL}(N, R)$ be a projective representation of a finite group $G$ and let $G$ act on $\mathbb{P}_{R}^{N-1}$ via $\rho$. Let $r \geqslant 1$ be an integer and suppose that the closed subscheme

$$
\Delta=\bigcup_{e \neq g \in G} \operatorname{Fix}(g)
$$

has closed fibre $\Delta_{k}$ of dimension at most $N-2-r$. Then there exist $G$-stable hypersurfaces $H_{1}, \ldots, H_{N-1-r}$ in $\mathbb{P}_{R}^{N-1}$ whose intersection $X$ is smooth of relative dimension $r$ over $R$ on which $G$ acts freely. Moreover, the hypersurfaces can be chosen so that the fibres of $X$ have ample canonical bundles.

Proof. We follow Serre's proof [Ser58] (also explained in [Ill05, Proposition 8.6.2]), except that now we work with $R$ (and not only with $k$ ) and that we allow $k$ to be finite by using [Gab01]. 


\section{PLURIGENERA OF GENERAL TYPE SURFACES IN MIXED CHARACTERISTIC}

First, let us consider the quotient scheme $Z:=\mathbb{P}_{R}^{N-1} / G$; it is also projective over $R$ by [EGAII Corollaire 6.6.4] and we fix a closed immersion $i$ of $Z$ into $\mathbb{P}^{M}$ over $R$ :

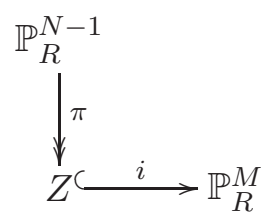

Then let us consider the base change by $R \longrightarrow k$. As $\Delta$ is projective over $R$, the restriction of $\pi$ to $\Delta$ induces a surjection, which we still denote by $\pi$, of $\Delta$ onto its scheme-theoretic image $\pi(\Delta)$ in $Z$; as surjectivity is stable under base change [EGAI, Proposition 3.5.2(ii)], $\Delta_{k} \subseteq \mathbb{P}_{k}^{N-1}$ surjects onto $(\pi(\Delta))_{k} \subseteq Z_{k}$ under $\pi_{k}$. Hence,

$$
\operatorname{dim}(\pi(\Delta))_{k} \leqslant \operatorname{dim}\left(\Delta_{k}\right) \leqslant(N-1)-1-r .
$$

By Bertini's theorem [Gab01, Corollary 1.7] applied to $(\pi(\Delta))_{k} \subseteq Z_{k}$ sitting in $\mathbb{P}_{k}^{M}$ via $i_{k}$, there exist hypersurfaces

$$
\left\{\overline{H_{i}^{\prime}}=\left(\overline{f_{i}^{\prime}}=0\right)\right\}_{i=1, \ldots, N-1-r}
$$

of respective degrees $d_{1}^{\prime}, \ldots, d_{N-1-r}^{\prime}$ in $\mathbb{P}_{k}^{M}$ such that: (a) their intersection $\overline{H^{\prime}}:=\overline{H_{1}^{\prime}} \cap \cdots \cap \overline{H_{N-1-r}^{\prime}}$ is disjoint from $i_{k}\left((\pi(\Delta))_{k}\right)$; and (b) $\overline{H^{\prime}} \cap i_{k}\left(Z_{k}\right)$ is smooth of dimension $r$ over $k$.

We then lift $\overline{f_{i}^{\prime}}$ arbitrarily to

$$
f_{i}^{\prime} \in \Gamma\left(\mathbb{P}_{R}^{M}, \mathcal{O}_{\mathbb{P}_{R}^{M}}\left(d_{i}^{\prime}\right)\right),
$$

set $H_{i}^{\prime}:=\left(f_{i}^{\prime}=0\right)$ in $\mathbb{P}_{R}^{M}$ for each $i$, and then put $H^{\prime}:=H_{1}^{\prime} \cap \cdots \cap H_{N-1-r}^{\prime}$. Then it follows that: (a) $H^{\prime} \cap i \pi(\Delta)=\varnothing$, for this intersection is a projective scheme over $R$ with empty special fibre; and (b) $H^{\prime} \cap i(Z)$ is smooth of relative dimension $r$ over $R$ (it is flat by [EGAIV, Proposition 0.15.1.16] and then smooth by [SGA1, II, Proposition 1.1 and Théorème 2.1], given that it is proper over $R$ ).

We note here that by using hypersurface sections of higher degree (which amounts to changing $i$ suitably), we can make the degrees $d_{i}^{\prime}$ as large as we want. Thus, we may assume that the resulting varieties have ample canonical bundles.

Now we return to the diagram $(2)$. As $(i \pi)^{*} \mathcal{O}_{\mathbb{P}_{R}^{M}}(1)$ is ample, it is isomorphic to $\mathcal{O}_{\mathbb{P}_{R}^{N-1}}(\delta)$ for some integer $\delta>0$. We pull back the sections $\left\{f_{i}^{\prime}\right\}$ by $i \pi$ :

$$
f_{i}:=(i \pi)^{*} f_{i}^{\prime} \in \Gamma\left(\mathbb{P}_{R}^{N-1}, \mathcal{O}_{\mathbb{P}_{R}^{N-1}}\left(d_{i}\right)\right),
$$

where we put $d_{i}:=\delta \cdot d_{i}^{\prime}$, and let

$$
H_{i}:=\left(f_{i}=0\right) \subseteq \mathbb{P}_{R}^{N-1}
$$

for each $i=1, \ldots, N-1-r$. Evidently, $H_{i}$ is invariant under $G$ for each $i$, and putting

$$
X:=H_{1} \cap \cdots \cap H_{N-1-r},
$$

one sees that $X$ is disjoint from $\Delta$.

\subsection{Some representations}

Let $e^{\prime} \geqslant 1$ be a given integer and put $G:=(\mathbb{Z} / p \mathbb{Z})^{\oplus e^{\prime}}$.

Let $A:=\mathbb{Z}[T] /\left(T^{p}-1\right)$ be the free $\mathbb{Z}$-module of rank $p$ on which multiplication by $T$ acts as an automorphism of exact order $p ; A$ is isomorphic to the group ring $\mathbb{Z}[\mathbb{Z} / p \mathbb{Z}]$. We denote this pair by $(A, T)$. Put $\ell=\ell(p, d):=\lceil(d+1) /(p-1)\rceil$ and $M:=A^{\oplus \ell}$ with the automorphism $T_{M}=T^{\oplus \ell}$, which we still denote by $T$. (The choice of $\ell$ will be justified by Lemma 2.2.1 later.) Finally,

$$
P_{e^{\prime}}:=M_{(1)} \oplus \cdots \oplus M_{\left(e^{\prime}\right)},
$$




\section{J. SuH}

where $M_{(i)}$ is a copy of $M$ for each $i=1, \ldots, e^{\prime}$. We denote by $T_{(i)}$ the automorphism of $P_{e^{\prime}}$ that acts as an identity on $M_{(j)}$ for $j \neq i$ and as $T$ on $M_{(i)}$. Then $T_{(i)}$ form a set of commuting automorphisms of exact order $p$.

Now we define a representation

$$
\widetilde{\rho}: G \longrightarrow \operatorname{GL}\left(e^{\prime} \cdot \operatorname{rank}_{\mathbb{Z}}(M), \mathbb{Z}\right)
$$

by mapping the element $(0, \ldots, 0,1,0, \ldots, 0)$ with the sole 1 in the $i$ th place to the element $T_{(i)}$ and then extending it $\mathbb{Z} / p \mathbb{Z}$-linearly. Then we denote by

$$
\rho: G \longrightarrow \operatorname{PGL}\left(e^{\prime} \cdot \operatorname{rank}_{\mathbb{Z}}(M), \mathbb{Z}\right)
$$

the composite of $\widetilde{\rho}$ with the natural morphism GL $\longrightarrow$ PGL. Put $m:=\operatorname{rank}_{\mathbb{Z}} M=\ell p$.

Lemma 2.2.1. Let $G$ act on $\mathbb{P}_{\mathbb{Z}}^{e^{\prime} m-1}$ via $\rho$. For $\underline{a}=\left(a_{1}, \ldots, a_{e}\right) \in G$ with exactly $n$ non-zero components, where $0<n \leqslant e^{\prime}$, the closed subscheme of fixed points of $\underline{a}$

$$
\operatorname{Fix}(\underline{a}) \subseteq \mathbb{P}_{\mathbb{Z}}^{e^{\prime} m-1}
$$

is contained in a closed subscheme $E_{\underline{a}}$ in $\mathbb{P}_{\mathbb{Z}}^{e^{\prime} m-1}$, flat of relative dimension

$$
\left(e^{\prime} m-1\right)-n(p-1) \ell
$$

over $\mathbb{Z}$. In particular, the locus on which $G$ does not act freely:

$$
\Delta=\bigcup_{0 \neq \underline{a} \in G} \operatorname{Fix}(\underline{a})
$$

has all fibres (over all prime fields) of dimension at most $\left(e^{\prime} m-1\right)-(d+1)$.

Proof. For any non-zero element $a \in \mathbb{Z} / p \mathbb{Z}$, one has $(M, T) \cong\left(M, T^{a}\right)$ as $\mathbb{Z}$-modules with automorphisms; therefore, one is reduced to the case where $\underline{a}$ is of the form $g_{n}:=(1, \ldots, 1,0, \ldots, 0)$ with $n$ 1s. Then the first assertion follows from the next lemma, and the last inequality follows from the choice of $\ell:(p-1) \ell \geqslant d+1$.

Lemma 2.2.2. Let $a \geqslant 1$ and $b \geqslant 0$ be integers and let $Q$ be the free $\mathbb{Z}$-module $A^{\oplus a} \oplus \mathbb{Z}^{\oplus b}$ with basis

$$
\left\{X_{(\alpha), i}: 1 \leqslant \alpha \leqslant a \text { and } i \in \mathbb{Z} / p \mathbb{Z}\right\} \cup\left\{Y_{j}: 1 \leqslant j \leqslant b\right\},
$$

where for each $\alpha$ and $i, X_{(\alpha), i}$ denotes the image of $T^{i}$ in the $\alpha$ th copy of $A=\mathbb{Z}[T] /\left(T^{p}-1\right)$. Consider the projective linear action of

$$
\phi=T^{\oplus a} \oplus 1_{\mathbb{Z} \oplus b}
$$

on $\mathbb{P}(Q)$. Then the subscheme $\operatorname{Fix}(\phi) \subseteq \mathbb{P}(Q)$ is contained in the subscheme $E$ of $\mathbb{P}(Q)$ defined by $a(p-1)$ equations

$$
\left(X_{(\alpha), i}\right)^{p}=\left(X_{(\alpha), i+1}\right)^{p}
$$

for each pair $(\alpha, i)$ with $1 \leqslant \alpha \leqslant a$ and $i \in\{0, \ldots, p-2\} \subseteq \mathbb{Z} / p \mathbb{Z}$.

Proof. We need to prove the inclusion of $S$-valued points: $\operatorname{Fix}(\phi)(S) \subseteq E(S)$ for any commutative ring $S$ with 1 . For this, we may assume that $S$ is local. Let

$$
\left[\cdots: x_{(\alpha), i}: \cdots: y_{j}: \cdots\right]
$$

be an $S$-valued point of $\operatorname{Fix}(\phi)$; that is, all the $x_{(\alpha), i}$ and $y_{j}$ are in $S$ and there exists a unit $\lambda \in S^{*}$ such that

This implies, in particular, that

$$
\phi(\underline{x}: \underline{y})=(\lambda \underline{x}: \lambda \underline{y}) .
$$

$$
x_{(\alpha), i}=\lambda x_{(\alpha), i+1} \quad \text { for each } \alpha \text { and every } i,
$$




\section{PLURIGENERA OF GENERAL TYPE SURFACES IN MIXED CHARACTERISTIC}

hence (by increasing $i$ by $p$ to come back),

$$
\left(1-\lambda^{p}\right) x_{(\alpha), i}=0 \quad \text { for all } \alpha, i \text {. }
$$

It then follows

$$
\left(x_{(\alpha), i}\right)^{p}=\left(\lambda x_{(\alpha), i+1}\right)^{p}=\lambda^{p}\left(x_{(\alpha), i+1}\right)^{p}=\left(x_{(\alpha), i+1}\right)^{p} .
$$

The rational map from $\mathbb{P}(Q)$ to $\mathbb{P}\left(\mathbb{Z}^{\oplus a} \oplus \mathbb{Z}^{\oplus b}\right)$ mapping $\left[\left(x_{(\alpha), i}\right)_{\alpha, i}:\left(y_{j}\right)_{j}\right]$ to $\left[\left(x_{(\alpha), 0}\right)_{\alpha}:\left(y_{j}\right)_{j}\right]$, restricts to a finite morphism on $E$. Thus, $E$ is flat of relative dimension at most $a+b-1$ over $\mathbb{Z}$.

\subsection{Proof of Theorem 0.1}

Now we prove Theorem 0.1 . Given $R, d \geqslant 2$ and $e \geqslant 1$, we choose $e^{\prime}$ to be equal to $e$ if $d=2$ and large enough so that $G_{d}\left(e^{\prime}\right) \geqslant e$ if $d \geqslant 3$. We take the module $P_{e^{\prime}} \otimes_{\mathbb{Z}} R$ and the associated projective space $\mathbb{P}$ on which $G=(\mathbb{Z} / p \mathbb{Z})^{\oplus e^{\prime}}$ acts projective linearly. Then Lemma 2.2.1 says that the pair $(\mathbb{P}, G)$ satisfies the condition of Lemma 2.1.1 with $r=d$. Thus, we obtain a smooth complete intersection $X / R$; that it has geometrically connected fibres with trivial $H^{i}(\mathcal{O})$ for $0<i<d$ follows from [SGA7, XI, Théorème 1.5]. Hence Lemma 1.1.1 applies and the resulting quotient $Y / R$ has

$$
p_{g}\left(Y_{k}\right)=p_{g}\left(Y_{K}\right)+G_{d}\left(e^{\prime}\right) .
$$

Note that when $d=2, G_{2}\left(e^{\prime}\right)=e$.

\subsection{Kodaira dimension and plurigenera of the examples}

In this section, we analyze the Kodaira dimension of the examples and show that they have ample canonical bundles. We analyze the exceptional cases, where the Kodaira dimension can be zero, in the next section.

Lemma 2.4.1. The value of $\delta$ in the proof of Lemma 2.1.1, when applied to our example for $e^{\prime} \geqslant 1$, is divisible by $p$ (and, hence, is at least $p$ ).

Proof. By passing to an algebraic closure if necessary, we may assume that $k$ is algebraically closed and we dispose of sufficiently many hyperplanes. Then considering $N-1$ hyperplanes of Bertini type in $\mathbb{P}_{k}^{M}$, and then comparing the intersection numbers, one deduces

$$
\delta^{N-1}=\operatorname{deg}\left(i_{k}, Z_{k}, \mathbb{P}_{k}^{M}\right) \cdot \operatorname{deg} \pi_{k}=\operatorname{deg}\left(i_{k}, Z_{k}, \mathbb{P}_{k}^{M}\right) \cdot p^{e^{\prime}} .
$$

Corollary 2.4.2. If $e^{\prime} \geqslant 2$ or if $e^{\prime}=1$ and $(d+1) /(p-1)$ is not an integer, then the resulting example $Y / R$ necessarily has ample canonical bundle.

Proof. It suffices to prove that $\omega_{X / R}=\pi^{*} \omega_{Y / R}$ is ample [EGAII, Corollaire 6.6.3]. We have

$$
\omega_{X / R} \cong \mathcal{O}_{X}\left(-e^{\prime} \ell p+\sum_{i=1}^{e^{\prime} \ell p-d-1} d_{i}\right)
$$

(to prove this formula, take the highest exterior power of the short exact sequence in [SGA7, XI, Equation 1.3.1] and see [SGA7, XI, §1.4]) and, by the previous lemma,

$$
-e^{\prime} \ell p+\sum_{i=1}^{e^{\prime} \ell p-d-1} d_{i} \geqslant-e^{\prime} \ell p+\left(e^{\prime} \ell p-d-1\right) p=e^{\prime}(p-1) \ell p-(d+1) p .
$$

This is positive in the two cases, because of the choice of $\ell$.

Corollary 2.4.3. Except possibly in the special case $e^{\prime}=1$ and $p-1 \mid d+1$, there exists an integer $m_{0}=m_{0}(Y)$ such that

$$
P_{m}\left(Y_{K} / K\right)=P_{m}\left(Y_{k} / k\right) \text { for all } m \geqslant m_{0} \text {. }
$$




\section{J. SuH}

Proof. As $\omega_{Y}$ is ample, by Serre's theorem [EGAIII, Théorème 2.2.1], there exists an integer $m_{0}$ such that all the higher cohomology groups of $\omega^{\otimes m}$ vanish for $m \geqslant m_{0}$. Then the invariance of Euler characteristic gives the desired equality. See also Theorem 1.2.1, where one can take $m_{0}:=2$.

\subsection{Exceptional cases}

Finally, let us place ourselves in the possible exceptional case; that is, in addition to $e^{\prime}=1$ and $p-1 \mid d+1$, let us assume that we can take $\delta$ in Lemma 2.1.1 equal to $p$ (smallest possible) so that $\omega_{X / R} \cong \mathcal{O}_{X}$. We do not know whether this is indeed possible.

If $p$ is odd, $(p-1) \mid(d+1)$ implies that $d$ is odd. Then $\operatorname{dim}_{k} H^{i}(\mathbb{Z} / p \mathbb{Z}, k)=1$ for every $i \geqslant 0$, and $\sum_{i=1}^{d-1}(-1)^{i} \operatorname{dim}_{k} H^{i}(\mathbb{Z} / p \mathbb{Z}, k)=0$, which means there is no jump in geometric genus. (Thus, in dimension at least three, the presence of infinitesimal torsion in the Picard scheme does not necessarily result in the gap in geometric genus.)

So let us turn to the case $p=2$. Again, if $d$ is odd, $p_{g}\left(Y_{k}\right)=p_{g}\left(Y_{K}\right)$, so let $d$ be even. In this case $\ell=\ell(p, d)=(d+1) /(2-1)=d+1$, and $X$ is a smooth complete intersection of $d+1$ quadrics in $\mathbb{P}_{R}^{2 d+1}$. The canonical bundles $\omega_{X_{K} / K}$ and $\omega_{X_{k} / k}$ are trivial, and as $\operatorname{Pic}^{\tau}(Y / R) \cong \mu_{2}$ (the Cartier dual of $\mathbb{Z} / 2)$, it follows that $\omega_{Y_{K} / K}^{\otimes 2}$ and $\omega_{Y_{k} / k}^{\otimes 2}$ are trivial.

On the other hand, we calculate that $\chi\left(X, \mathcal{O}_{X}\right)=2$, hence $\chi\left(Y, \mathcal{O}_{Y}\right)=1$. As $\operatorname{dim}_{k} H^{i}(\mathbb{Z} / 2 \mathbb{Z}, k)=$ 1 for $i \geqslant 0$ and $H^{i}(\mathbb{Z} / 2 \mathbb{Z}, K)=0$ for $i>0$, it follows that $p_{g}\left(Y_{K} / K\right)=\operatorname{dim}_{K} H^{0}\left(Y_{K}, \omega_{Y_{K} / K}\right)=0$ and $p_{g}\left(Y_{k} / k\right)=\operatorname{dim}_{k} H^{0}\left(Y_{k}, \omega_{Y_{k} / k}\right)=1$. Therefore, we have

$$
\omega_{Y_{k} / k} \cong \mathcal{O}_{Y_{k}}, \quad \omega_{Y_{K} / K} \approx \mathcal{O}_{Y_{K}} \quad \text { and } \quad \omega_{Y_{K} / K}^{\otimes 2} \cong \mathcal{O}_{Y_{K}} .
$$

These imply

$$
\begin{aligned}
P_{2 m}\left(Y_{k} / k\right) & =1=P_{2 m}\left(Y_{K} / K\right), \\
P_{2 m+1}\left(Y_{k} / k\right) & =1=P_{2 m+1}\left(Y_{K} / K\right)+1,
\end{aligned}
$$

for all $m \geqslant 0$. We note that in case $d=2, X / R$ is a smooth intersection of three quadrics in $\mathbb{P}^{5}$, that is, a K3 surface, and that $Y / R$ is the quotient of $X / R$ by a free $\mathbb{Z} / 2$-action, that is, an Enriques surface.

\section{Quaternionic and unitary Shimura surfaces}

We consider certain compact Shimura varieties obtained as quotients, either of the two-fold product of the Poincaré upper half-plane by irreducible lattices in $\mathrm{SL}_{2}(\mathbb{R}) \times \mathrm{SL}_{2}(\mathbb{R})$, or of the complex twodimensional unit ball by lattices in $\mathrm{SU}(1,2)$. In both cases, global sections of the canonical bundle can be interpreted as holomorphic modular forms.

These surfaces are canonically defined over number fields, and have integral models for all but finitely many primes. We exhibit cases where the special fibre has larger geometric genus than the generic fibre; in such cases, we can say that we have more modular forms modulo $p$.

First, we set up the situation and recall some facts.

\subsection{Shimura data}

We will be in one of the following three situations. We refer to [Rei97] and [Kot92] for details.

3.1.1 Quaternionic case. Let $F$ be a totally real number field of degree $d \geqslant 2$ over $\mathbb{Q}$, and let $D$ be a central division algebra of dimension four over $F$ such that $D \otimes_{\mathbb{Q}} \mathbb{R}$ is isomorphic to the product of two copies of $M_{2}(\mathbb{R})$ and $d-2$ copies of the Hamilton's quaternion algebra $\mathbb{H}$. 


\section{PluRigenera of General type SURFACES IN MIXED CHARACTERISTIC}

Define the $\mathbb{Q}$-algebraic group $G:=D^{\times}$by

$$
D^{\times}(R)=\left(D \otimes_{\mathbb{Q}} R\right)^{\times},
$$

for any $\mathbb{Q}$-algebra $R$. Denoting $\mathbb{S}:=\operatorname{Res}_{\mathbb{C} / \mathbb{R}} \mathbb{G}_{m}$ and using the decomposition of $D \otimes \mathbb{R}$ as above, we have the conjugacy class $X$ of the homomorphism $h: \mathbb{S} \longrightarrow G_{\mathbb{R}}$ mapping $z=a+b i \in \mathbb{C}^{\times}$to $\left(\begin{array}{cc}a & b \\ -b & a\end{array}\right)$ (respectively to 1 ) in the factor $G L_{2}(\mathbb{R})$ (respectively $\mathbb{H}^{\times}$). Thus, we have a Shimura datum $(G, X)$.

3.1.2 Unitary case $A_{1} \times A_{1}$. Let $F^{+}$be a totally real number field of degree $d \geqslant 2$ over $\mathbb{Q}, F$ a quadratic totally imaginary extension of $F^{+}$, and $D$ a central division algebra of dimension four over $F$ with a positive involution $*$ inducing complex conjugation on $F$. Considering $V=D$ as a left $D$-module, let

$$
\psi: V \times V \longrightarrow \mathbb{Q}
$$

be an alternating $D$-hermitian (meaning $\psi(b u, v)=\psi\left(u, b^{*} v\right)$ ) bilinear form.

The pairing $\psi$ induces an involution $*_{\psi}$ on $C:=\operatorname{End}_{D}(V)$; let $h: \mathbb{C} \longrightarrow C_{\mathbb{R}}$ be an $\mathbb{R}$-algebra homomorphism such that (i) $h$ transforms complex conjugation on $\mathbb{C}$ into $*_{\psi}$ on $C_{\mathbb{R}}$ and (ii) the symmetric $\mathbb{R}$-bilinear form $(u, v) \mapsto \psi(u, h(i) v)$ on $V_{\mathbb{R}}$ is positive definite.

We define the $\mathbb{Q}$-algebraic group $G$ by

$$
G(R)=\left\{g \in C \otimes_{\mathbb{Q}} R: g g^{*} \in R^{\times}\right\},
$$

for any $\mathbb{Q}$-algebra $R$, and assume that $G_{\mathbb{R}}^{\text {der }}$ is isomorphic to the product of two copies of $\mathrm{SU}(1,1)$ and $d-2$ copies of $\mathrm{SU}(2)$. Then the $G(\mathbb{R})$-conjugacy class $X$ of the induced group homomorphism $h: \mathbb{S} \longrightarrow G_{\mathbb{R}}$ defines a Shimura datum $(G, X)$ (see $\left.[\operatorname{Kot} 92, \S 5]\right)$.

3.1.3 Unitary case $A_{2}$. Let $F^{+}$be a totally real number field of degree $d \geqslant 1$ over $\mathbb{Q}, F$ a quadratic totally imaginary extension of $F^{+}$, and $D$ a central division algebra of dimension nine over $F$. Let $*, V, \psi$ and $h$ be as in the case above, with $C$ and $G$ defined similarly.

We assume, in this case, that $G_{\mathbb{R}}^{\text {der }}$ is isomorphic to the product of one copy of $\operatorname{SU}(1,2)$ and $d-1$ copies of SU(3). This again defines a Shimura datum $(G, X)$ (see $[\operatorname{Kot} 92, \S 5])$.

3.1.4 Canonical models and connected components. Given a Shimura datum $(G, X)$ as above, the Shimura varieties

$$
S h_{K}(G, X):=G(\mathbb{Q}) \backslash X \times G\left(\mathbb{A}_{f}\right) / K,
$$

as $K$ ranges over open compact subgroups of $G\left(\mathbb{A}_{f}\right)$, are canonically defined over a number field $E(G, X)$ called the reflex field. The variety $S h_{K}(G, X)$ is a finite disjoint union of varieties of the form $X_{\Gamma}^{+}:=X^{+} / \Gamma$, where $X^{+}$is a connected component of $X$ and $\Gamma$ is a congruence subgroup of $G^{\text {der }}(\mathbb{Q})$. Each $X_{\Gamma}^{+}$is then defined over an abelian extension (depending on $K$ ) of $E(G, X)$, specified by the reciprocity map. When $\Gamma$ is torsion-free, $X_{\Gamma}^{+}$is a smooth variety, and an inclusion $\Gamma_{1} \subseteq \Gamma$ of congruence subgroups induces a finite étale morphism $X_{\Gamma_{1}}^{+} \longrightarrow X_{\Gamma}^{+}$, defined over an abelian extension of $E(G, X)$.

We note that the derived group $G^{\text {der }}$ is simply connected and that $S h_{K}(G, X)$ is projective in all of the three cases.

\subsection{Integral models and the set of good primes}

In both of the unitary cases as well as in the quaternionic case with $d=2$ (so that $D \otimes_{\mathbb{Q}} \mathbb{R}$ is totally indefinite), the Shimura variety attached to $(G, X)$ has a PEL modular interpretation, and has integral canonical models [Kot92, Moo98]. For models in the quaternionic case with $d>2$, see [Rei97, $\S \S 1-6]$. 


\section{J. SuH}

We use the following fact: we have a finite set $\Sigma$ of rational primes $p$ and (hyperspecial) open compact subgroups $K_{p}$ of $G\left(\mathbb{Q}_{p}\right)$ for each $p$ outside $\Sigma$, such that whenever $K^{p}$ is a sufficiently small compact open subgroup of $G\left(\mathbb{A}_{f}^{p}\right)$ :

(i) $S h_{K_{p} K^{p}}$ has a smooth projective model $\mathcal{M}_{K_{p} K^{p}}$ over $\mathcal{O}_{E(G, X)} \otimes \mathbb{Z}_{(p)}$;

(ii) the connected components of $S h_{K_{p} K^{p}}$ are defined over an abelian extension of $E(G, X)$, which is unramified above $p$ (see [Moo98, Lemma 3.15]); and

(iii) for an open compact subgroup $K_{1}^{p} \subseteq K^{p}$, the transition morphism extends to a finite étale morphism $\mathcal{M}_{K_{p} K_{1}^{p}} \longrightarrow \mathcal{M}_{K_{p} K^{p}}$.

For the explicit description of $\Sigma$, we refer to [Rei97, §6] and [Kot92, §5].

\subsection{Statement of theorem}

Now let $N=p_{1} \cdots p_{n}$ be a given product of distinct rational primes outside $\Sigma$ and $e \geqslant 1$ a given integer. Denote by $\mathbb{A}_{f}^{N}:=\mathbb{Q} \otimes \prod_{p \nmid N} \mathbb{Z}_{p}$ the ring of finite adeles away from $N$ and put $K_{N}:=K_{p_{1}} \cdots K_{p_{n}}$. For a sufficiently small open compact subgroup $K^{N}$ of $G\left(\mathbb{A}_{f}^{N}\right)$, any connected component $Y$ of $S h_{K_{N} K^{N}}$ is defined over an abelian extension $E_{Y}$ of $E(G, X)$ and has an integral model $\mathcal{Y}_{\wp}$ over $\left(\mathcal{O}_{E_{Y}}\right)_{\wp}$, for each prime $\wp$ of $E_{Y}$ dividing $N$. Then $\mathcal{Y}_{\mathbb{C}}$ is of the form $X_{\Gamma}^{+}$, where $X^{+}$is a connected component of $X$ and $\Gamma$ is a congruence subgroup of $G^{\text {der }}(\mathbb{Q})$. One writes $\Gamma=G^{\text {der }}(\mathbb{Q}) \cap C$, where $C=C_{N} C^{N}$ is a compact open subgroup of $G^{\operatorname{der}}\left(\mathbb{A}_{f}\right)=G^{\operatorname{der}}\left(\prod_{p \mid N} \mathbb{Q}_{p}\right) \cdot G^{\operatorname{der}}\left(\mathbb{A}_{f}^{N}\right)$.

Theorem 3.4. Fix $(G, X)$ and $\Sigma$ as in the previous sections, and let $N, e, Y$ be given as above. Then there exists an open subgroup $C_{1}^{N}$ of $C^{N}$ such that the corresponding $Y_{1} / E_{Y_{1}}$ and $\mathcal{Y}_{1, \wp}$ have

$$
p_{g}\left(\mathcal{Y}_{1, \wp} \otimes \kappa(\wp)\right) \geqslant p_{g}\left(Y_{1}\right)+e
$$

for every prime $\wp$ of $E_{Y_{1}}$ dividing $N$.

Proof. We find subgroups $C_{0}^{N} \subseteq C_{1}^{N}$ in $C^{N}$ such that the resulting congruence subgroups $\Gamma_{i}:=$ $G^{\mathrm{der}}(\mathbb{Q}) \cap C_{i}^{N} C_{N}$ for $i=0,1$ satisfy

$$
\Gamma_{1} / \Gamma_{0} \cong C_{1}^{N} / C_{0}^{N} \cong(\mathbb{Z} / N \mathbb{Z})^{e} ;
$$

the first isomorphism follows from the strong approximation theorem.

Let us first assume that we have located these subgroups. Then, in the quaternionic case and the unitary case $A_{1} \times A_{1}, \Gamma_{i}$ define irreducible lattices in $\mathrm{SL}_{2}(\mathbb{R}) \times \mathrm{SL}_{2}(\mathbb{R})$, and hence $\Gamma_{i}^{\mathrm{ab}}=\Gamma_{i} /\left[\Gamma_{i}, \Gamma_{i}\right]$ is finite. Putting $Y_{i}:=X_{\Gamma_{i}}^{+}$for $i=0,1$, one deduces $H^{1}\left(Y_{i}, \mathcal{O}_{Y_{i}}\right)=0$. The unitary case $A_{2}$ is much more subtle, for $\mathrm{SU}(1,2)$ has real rank one and does admit cocompact congruence subgroups with non-zero first Betti number [Kaz77, §4]. However, our choice of $G$ (and hence of $\Gamma_{i}$ ) was made precisely so that [Rog90, Theorem 15.3.1] (for $d=1$ ) and [Clo93, Theorem 3.2(i)] (for $d \geqslant 1$ ) apply, from which we deduce $H^{1}\left(Y_{i}, \mathcal{O}_{Y_{i}}\right)=0$ for $i=0,1$ (see also [Sar05]).

Then we apply Lemma 1.1.1 to the integral model of $Y_{1}$, which has a connected finite étale Galois covering with group $(\mathbb{Z} / N \mathbb{Z})^{\oplus e}$, hence also one with group $\left(\mathbb{Z} / p_{i} \mathbb{Z}\right)^{\oplus e}$ for each $p_{i}$ dividing $N$.

It remains to construct the subgroups. First by shrinking $C^{N}$ if necessary, we may assume that it is of the form

$$
C^{N}=\prod_{p \nmid N} C_{p},
$$

where $C_{p}$ are open compact subgroups of $G^{\mathrm{der}}\left(\mathbb{Q}_{p}\right)$. Then there are infinitely many prime numbers $\ell$ outside $\Sigma$ such that $(\mathrm{i}) \ell \equiv 1(\bmod N)$ and (ii) $C_{\ell} \cong \mathrm{SL}_{2}\left(\mathbb{Z}_{\ell}\right)^{d}\left(\right.$ respectively $\left.C_{\ell} \cong \mathrm{SL}_{3}\left(\mathbb{Z}_{\ell}\right)^{d}\right)$ in the quaternionic case and the unitary case $A_{1} \times A_{1}$ (respectively in the unitary case $A_{2}$ ). We take $M:=e \cdot n$ of them, say $\left\{\ell_{1}, \ldots, \ell_{M}\right\}$, and let $C_{0, \ell_{i}}$ be the subgroup of $C_{\ell_{i}}$ consisting of the elements 


\section{PLURIGENERA OF GENERAL TYPE SURFACES IN MIXED CHARACTERISTIC}

congruent to 1 modulo $\ell_{i}$. We let

$$
C_{0}^{N}:=\prod_{i=1}^{M} C_{0, \ell_{i}} \times \prod_{\left(p, N \ell_{1} \cdots \ell_{M}\right)=1} C_{p} .
$$

We note that the cardinalities of $\mathrm{SL}_{2}\left(\mathbb{F}_{\ell}\right)^{d}$ and $\mathrm{SL}_{3}\left(\mathbb{F}_{\ell}\right)^{d}$ are divisible by $N$, hence by each $p_{i}$, for $\ell=\ell_{1}, \ldots, \ell_{M}$. Thus, there exists a subgroup of $C^{N} / C_{0}^{N}$ that is isomorphic to $(\mathbb{Z} / N \mathbb{Z})^{\oplus e}$, which corresponds to the desired subgroup $C_{1}^{N}$, sitting between $C^{N}$ and $C_{0}^{N}$, with $C_{1}^{N} / C_{0}^{N} \cong(\mathbb{Z} / N \mathbb{Z})^{e}$.

Remark 3.5. The canonical number fields over which the models are defined are unramified over any rational prime dividing $N$, as noted above. Moreover, these surfaces have ample canonical bundles. Therefore, it follows from Theorem 1.2.1(ii) that their higher plurigenera $\left(P_{m}\right.$ with $\left.m \geqslant 2\right)$ are invariant under reduction.

\section{ACKNOWLEDGEMEnTs}

This article is essentially one third of my $\mathrm{PhD}$ thesis at Princeton University; I thank my advisor Nicholas Katz for his help and encouragement. Max Lieblich suggested the use of Kodaira vanishing as in [DI87]; I learned relevant facts about lattices in $\mathrm{SU}(1,2)$ from Peter Sarnak and Hee Oh; Luc Illusie pointed out that the construction of complete intersection with group actions had been considered earlier by Raynaud; and I benefited from conversations with János Kollár and Minhyong Kim. I sincerely thank them all.

\section{REFERENCES}

AM94 A. Adem and R. Milgram, Cohomology of finite groups, Grundlehren der Mathematischen Wissenschaften, vol. 309 (Springer, Berlin, 1994).

Clo93 L. Clozel, On the cohomology of Kottwitz's arithmetic varieties, Duke Math. J. 72 (1993), 757-795.

DI87 P. Deligne and L. Illusie, Relèvement modulo $p^{2}$ et décomposition du complexe de Rham, Invent. Math. 89 (1987), 247-270.

EGAI A. Grothendieck, Eléments de géométrie algébrique. I. Le langage des schémas, Pub. Math. Inst. Hautes Études Sci. 4 (1960).

EGAII A. Grothendieck, Eléments de géométrie algébrique. II. Etude globale élémentaire de quelques classes de morphismes, Pub. Math. Inst. Hautes Études Sci. 8 (1961).

EGAIII A. Grothendieck, Eléments de géométrie algébrique. III. Etude cohomologique des faisceaux cohérents. I, Pub. Math. Inst. Hautes Études Sci. 11 (1961); II, idem. 17 (1963).

EGAIV A. Grothendieck, Eléments de géométrie algébrique. IV. Etude locale des schémas et des morphismes de schémas. I, Pub. Math. Inst. Hautes Études Sci. 20 (1964); II, idem. 24 (1965); III, idem. 28 (1966); IV, 32 (1967).

Gab01 O. Gabber, On space filling curves and Albanese varieties, Geom. Funct. Anal. 11 (2001), 1192-1200.

Gro62 A. Grothendieck, Fondements de la géométrie algébrique. VI. Les schémas de Picard: propriétés générales, Extraits du Séminaire Bourbaki (1957-1962), Exposé 236 (Secrétariat mathématique, Paris, 1962).

Ill05 L. Illusie, Grothendieck's existence theorem in formal geometry (with a letter of J.-P. Serre), Fundamental algebraic geometry, Mathematical Surveys and Monographs, vol. 123 (American Mathematical Society, Providence, RI, 2005), 179-233.

Kaz77 D. Kazhdan, Some applications of the Weil representation, J. Anal. Math. 32 (1977), 235-248.

Kot92 R. Kottwitz, Points on some Shimura varieties over finite fields, J. Amer. Math. Soc. 5 (1992), $373-444$. 


\section{PLURIGENERA OF GENERAL TYPE SURFACES IN MIXED CHARACTERISTIC}

KU85 T. Katsura and K. Ueno, On elliptic surfaces in characteristic p, Math. Ann. 272 (1985), 291-330.

Lan83 W. Lang, On Enriques surfaces in characteristic p. I, Math. Ann. 265 (1983), 45-65.

Moo98 B. Moonen, Models of Shimura varieties in mixed characteristics, in Galois representations in arithmetic algebraic geometry (Durham, 1996), London Mathematical Society Lecture Note Series, vol. 254 (Cambridge University Press, Cambridge, 1998), 267-350.

Ray79 M. Raynaud, "p-torsion" du schéma de Picard in Journées de Géométrie Algébrique de Rennes (Rennes, 1978), Vol. II, Astérisque, vol. 64 (Societé Mathématique de France, Paris, 1979), $87-148$.

Rei97 H. Reimann, The semi-simple zeta function of quaternionic Shimura varieties, Lecture Notes in Mathematics, vol. 1657 (Springer, Berlin, 1997).

Rog90 J. Rogawski, Automorphic representations of unitary groups in three variables, Annals of Mathematics Studies, vol. 123 (Princeton University Press, Princeton, NJ, 1990).

Sar05 P. Sarnak, Notes on the generalized Ramanujan conjectures, in Harmonic analysis, the trace formula, and Shimura varieties, Clay Mathematics Proceedings, vol. 4 (American Mathematical Society, Providence, RI, 2005), 659-685.

Ser58 J.-P. Serre, Sur la topologie des variétés algébriques en caractéristique p, in 1958 Symposium internacional de topología algebraica (International symposium on algebraic topology) (Universidad Nacional Autónoma de México and UNESCO, Mexico City), 24-53.

SGA1 A. Grothendieck, Revêtements étales et groupe fondamental, Séminaire de géométrie algébrique du Bois-Marie 1960-1961 (SGA1). With two papers by M. Raynaud. Lecture Notes in Mathematics, vol. 224 (Springer, Berlin, 1971).

SGA7 A. Grothendieck, P. Deligne and N. Katz, Groupes de monodromie en géométrie algébrique. I, Séminaire de géométrie algébrique du Bois-Marie 1967-1969 (SGA7), Lecture Notes in Mathematics, vol. 288; II, idem. vol. 340 (Springer, Berlin, 1972-1973).

Junecue Suh junecuesuh@math.mit.edu

Department of Mathematics, Massachusetts Institute of Technology, Cambridge, MA 02139, USA 